\title{
Crystallization medium variations as a factor of oxalate patina formation
}

\author{
Aleksei Victorovich Rusakov ${ }^{1}$, Maria Kuzmina ${ }^{1}$, Olga Frank-Kamenetskaya ${ }^{1}$, Dmitri Vlasov ${ }^{1}$ \\ ${ }^{1}$ Saint Petersburg State University Institue Of Earth Sciences, Saint Petersburg, Russian Federation \\ E-mail: alex.v.rusakov@gmail.com
}

Biofilms containing colonies of microscopic fungi (micromicetes) can often be found on the surface of carbonate rocks (marble, limestone etc.) in urban environment. Micromycetes excrete substantial amounts of organic acids (oxalic, citric, malic, succinic, fumaric, lactic etc. [1]) in the course of their metabolism. These acids solubilize carbonate rocks and induce calcium oxalate (calcium oxalate monohydrate - whewellite, calcium oxalate dihydrate - weddellite) crystallization. These minerals as well as micromicete metabolites and several other chemical impurities which come from the environment form the so called oxalate patina. The influence of oxalate patina on the surface of carbonate monuments is ambiguous because the mechanisms of its formation are not yet fully known. The aim of this work was to study the influence of different organic and inorganic ions found in biofilms [2] on mineral composition and the morphology of the formed crystals (mostly weddellite and whewellite) in vitro.

Calcium oxalate crystallization was performed by decantation of a mixture of sodium oxalate and calcium chloride aqueous solutions with $\mathrm{pH}$ variation (4.0-7.0) at room temperature (22-25C). The precipitate was filtered, washed with distilled water and dried in air at room temperature. $\mathrm{Na}+, \mathrm{K}+, \mathrm{Mg} 2+, \mathrm{Fe} 3+, \mathrm{PO} 43-, \mathrm{SO} 42-$ ions were added to the solution as the impurity components as well as the organic acids, commonly excreted by micromicetes [1], the organic acid concentrations were close to the ones observed in the biofilms.

It was found that whewellite crystallizes in the presence of all inorganic and organic components in any given concentrations in the form of small spherulites - white beads without crystalline faces.

Weddellite formed in the presence of citric ions of varying concentrations with no dependance on the presence of inorganic components, as well as in the presence of three acids (malic, succinic and fumaric) with an equal ratio of their concentrations in the absence of inorganic components (Fig. 1). Weddellite solely formed when $\mathrm{Ca} 2+$ ions to the oxalate ions concentration was set close to stoichiometry $(2.5: 1)$ at $\mathrm{pH} \geq 6$, and at an increased ratio of more than $5.5: 1$ at $\mathrm{pH} \geq 5$. Weddellite crystals had tetragonal dipyramid facetes, and with the increase of $\mathrm{Ca} 2+$ concentration (while C2O42- concentration was set to 3 $\mathrm{mmol} / \mathrm{I}$ ) a transition from dipyramidal to skeletal crystals was observed. The formation of prism facets of weddellite crystals was observed only in the presence of citrate ions and MgSO4 in the solution.

Calcium oxalate trihydrate (caoxite, not yet found in biofilms) formed in small amounts in the presence of fumaric or succinic acids along with whewellite crystals, and in the presence of citric acid the impurities of MgSO 4 and Fe3+.

The results contribute to the research of the oxalate patina formation and give evidence on the specific patina components which influence the mineral composition and the crystal morphology.

The research was partially supported by the Russian Foundation for Basic Research (Project 16-05-00986-a) and Saint Petersburg State University project 3.38.243.2015 on the stages of experimental data collection and analysis.

[1] Sazanova, K.V. et al. (2014) Microbiology. 83-5, 516-522.

[2] Frank-Kamenetskaya, O.V. et al. (2009) Studia Univ. Babes-Bolyai. Geologia, 54-2, 17-22.

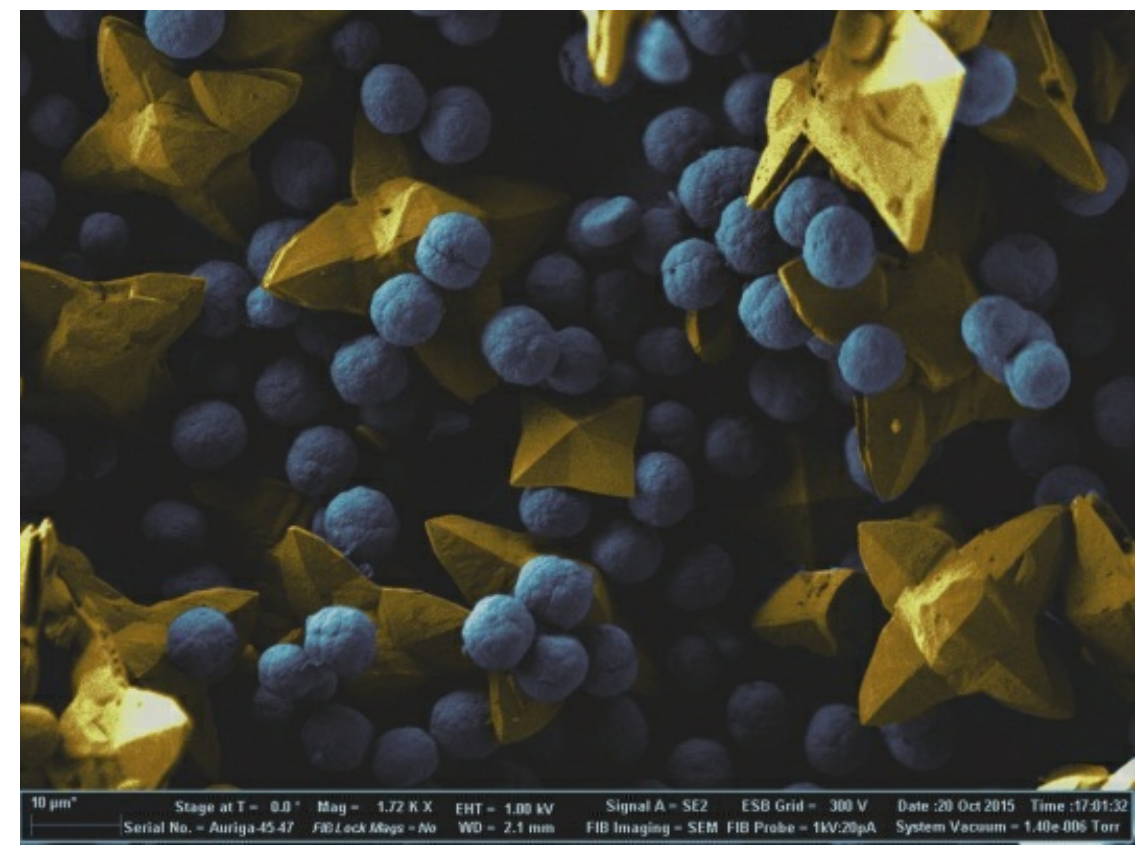

Keywords: calcium oxalates, oxalate patina, micromicetes 